\title{
Combination of bacteriolytic therapy with magnetic field for Ehrlich tumour treatment
}

\author{
Fadel M. Ali, Reem H. El-Gebaly and Amany M. Hamad \\ Biophysics Department, Faculty of Science, Cairo University, Giza, Egypt
}

\begin{abstract}
Referable to the limited response of the current available cancer treatment modalities, new effective cancer fighting treatments are needed. This work investigates the efficiency of intratumoural injection of Pseudomonas aeruginosa bacteria combined with a local tumour exposure to extremely low frequency square pulsed magnetic field (ELF SPMF) in the mouse Ehrlich tumour. 64 Ehrlich ascites tumour-implanted female albino BALB/C mice were equally split up into 4 groups. Group 1 (GP1) was the positive control group. Group 2 (GP2) received a single intratumoural injection of $P$. aeruginosa bacteria. Group 3 (GP3) was exposed to ELF SPMF for tumour local exposure. Group 4 (GP4) was treated with P. aeruginosa intratumoural injection followed by local exposure of the tumour to ELF SPMF. Treatment monitoring was evaluated using ultrastructural examination, flow cytometry analysis in addition to the measurement of tumour dielectric properties. Tumour cell apoptosis was obvious in GP2 and GP4, but, with higher severity and percentages in GP2. Tumour biophysical properties revealed a significant increase in static conductivity $\sigma_{\mathrm{S}}$ of GP2 , and decreases in dielectric increment $\Delta \varepsilon^{\prime}$ of both GP2 and GP4 compared to the GP1. Unfortunately, GP2 mice showed severe signs of toxicity. We advocate the utilization of the combination of P. aeruginosa and SPMF to yield the most effective antitumour agent with less bacteria-related toxicity.
\end{abstract}

Key words: Ehrlich tumour - P. aeruginosa - Bacteriolytic therapy - ELF SPMF - Cytotoxicity - Apoptosis - Dielectric properties

\section{Introduction}

Cancer is among the major causes of mortality and morbidity worldwide. Currently traditional anticancer therapies that include surgery mostly followed by chemotherapy and/ or radiotherapy are often associated with severe toxic side effects. Therefore, there are different trails for using alternative cancer treatment modalities; one of such methods is the microbial therapy.

Infecting or injecting mice with live attenuated Salmonella typhimurium cells could contribute to significant regression of tumours transplanted in mice (Pawelek et al. 1997). Furthermore, many live or attenuated pathogenic bacteria or their products have been applied in an attempt to reduce growth rate or size of tumours (Chakrabarty 2003; Theys et

Corresponding author: Reem H. El-Gebaly, Biophysics Department, Faculty of Science, Cairo University, Giza, Egypt

E-mail: r_elgebaly@msn.com al. 2003; Michl and Gress 2004; Maletzki et al. 2008; VázquezRivera et al. 2015; Kuan and Lee 2016).

Regardless the clinical importance of bacteria and their encouraging results as anticancer agents, the undesired systemic infections (i.e., toxicity) were a major drawback of their employment. Therefore, new techniques are urgently needed in order to defeat this problem based on reducing overall undesired toxicity to normal tissues. Nowadays, considerable efforts had been dedicated to investigate the effect of extremely low frequency electromagnetic fields (ELF EMFs) on bacterial growth in order to develop an alternative method for controlling bacterial infection (Strasák et al. 2002; Li et al. 2004; Segatore et al. 2012; Fadel et al. 2014).

Serag et al. (2014) proposed possible advancement for accelerating healing of wounds in guinea pigs infected with $P$. aeruginosa bacteria using the resonance-frequency phenomenon. The authors exposed the infected wounds to the square pulsed magnetic field of specific frequency $(0.7 \mathrm{~Hz})$. 
In a study reported by Oncul et al. (2016), the authors found alterations in the behavior of both Gram-positive and Gram-negative bacteria and in their physicochemical properties as well, after exposure to extremely low frequency electromagnetic field $(50 \mathrm{~Hz}, 1 \mathrm{mT}, 2 \mathrm{~h})$.

Among the results of a more recent work directed by Fadel et al. (2016), exposure of $P$. aeruginosa bacteria samples to positive electric pulses at the same mentioned frequency $0.7 \mathrm{~Hz}$ caused a $47.4 \%$ reduction in its growth rate in comparison to unexposed one. The authors explained that this inhibitory effect was due to the exposed $P$. aeruginosa exhibited changes in DNA pattern and cell wall damages as noticed from TEM images.

The purpose of the present study was to examine the effect of live $P$. aeruginosa injected into experimentally implanted Ehrlich tumour mass in female mice. The aim extended also to control the bacterial cellular growth inside the tumour mass by exposure to extremely low frequency square pulsed magnetic field (ELF SPMF) at a resonance frequency $0.7 \mathrm{~Hz}$.

\section{Materials and Methods}

\section{Bacterial strain and media used}

The bacterial strain Pseudomonas aeruginosa ATCC 9027 used in the present study was previously isolated aseptically from the outer ear infection, and then transported to the Microbiological Resources Center (Cairo MIRCEN), Faculty of Agriculture, Ain Shams University, Egypt; to generate pure bacterial isolates. Nutrient Agar (NA) and Nutrient broth (NB) media used for cultivation of bacteria were purchased from Sigma-Aldrich Chemical Company (St. Louis, USA).

\section{Experimental animals}

This study was carried out on 64 female Albino BALB/C mice, each of $\sim 45$ days age and average body weight 27-30 g. The animals were kept under standard laboratory conditions 2 weeks for adaptation with a temperature around $23-25^{\circ} \mathrm{C}$. They were fed with a suitable quantity of complete mouse diet and tap water. All animal procedures and handling were carried out in accordance with the established guidelines for the Care and Use of Laboratory Animals (Guide for the Care and Use of Laboratory Animals ( $8^{\text {th }}$ edition), National Academies Press, Washington, D.C., published in 2011).

\section{Tumour implantation}

Ehrlich ascites carcinoma fluid was withdrawn into a sterile insulin disposable syringe fitted with a 29 1/2 -gauge needle (1-cc; Terumo, Tokyo), from the peritoneal cavity of a donor mouse on the $7^{\text {th }}$ day after injection, prepared in Cancer Biology Department of the National Cancer Institute (NCI), Cairo University, Egypt. The isolated ascites Ehrlich tumour cells were routinely diluted in sterile phosphate buffered saline (PBS, $\mathrm{pH}=7.4$ ) and then tested for its viability. After that, $0.2 \mathrm{ml}$ of the above suspension with concentration $\sim 1.0 \times 10^{6}$ tumour cells/ml was subcutaneously (s.c.) injected into the right thigh of the lower limb of each mouse. The day of tumour implantation was assigned as day zero.

\section{Experimental design}

The Ehrlich tumour-injected mice were equally divided into 4 groups ( $n=16$ mice/group) at day 8 post-tumour implantation. At day 13 post-tumour implantation, treatment begun for different group where tumours reached a size of $\sim 0.241 \mathrm{~cm}^{3}$, that was clearly visible. Mice of group 1 (GP1) were served as the positive control tumour-bearing group and were not subjected to any treatment during the course of the experiment. Mice of group 2 (GP2) were injected by one shot in the cortex of the Ehrlich tumour with $0.1 \mathrm{ml}$ of a suspension containing $\sim 1.5 \times 10^{8}$ CFUs (colony-forming units)/ml of $P$. aeruginosa bacteria and left without any further treatment. Mice of group 3 (GP3) were exposed once to SPMF at a magnetic flux density of $0.88 \pm 0.02 \mathrm{mT}$ and frequency $0.7 \mathrm{~Hz}$ for a period of one hour. Exposure was directed locally to the implanted tumour in the right thigh. Mice of group 4 (GP4) were injected by one shot in the cortex of the Ehrlich tumour with $0.1 \mathrm{ml}$ of a suspension containing $\sim 1.5 \times 10^{8} \mathrm{CFU} / \mathrm{ml}$ of $P$. aeruginosa. After two hours of infection, mice were locally exposed once at the palpable tumour site to SPMF at a magnetic flux density of $0.88 \pm 0.02 \mathrm{mT}$ and frequency $0.7 \mathrm{~Hz}$ for a period of one hour.

\section{Preparation and intratumoural administration of P. aeruginosa}

Two viable bacterial colonies from the pure isolated bacteria nutrient agar plate were inoculated in a $25 \mathrm{ml}$ flask containing $5 \mathrm{ml}$ of sterile nutrient broth (NB) medium of $\mathrm{pH} 7.4 \pm 0.2$. After inoculation, the bacterial culture was incubated at $37^{\circ} \mathrm{C}$ overnight. On the following day, the fresh culture of the tested bacteria was chilled by swirling the tube in ice water, and the bacterial cell concentration was adjusted to be equivalent to $0.5 \mathrm{McF}$ arland turbidity standard (McFarland 1907; Koneman et al. 1992) by performing dilutions until the culture reached an optical density $\left(\mathrm{OD}_{625 \mathrm{~nm}} \sim 0.1\right)$ using spectrophotometer type JENWAY 6405 UV/Visible-UK. Afterwards, the mice were taken to the laminar air flow unit, and $0.1 \mathrm{ml}$ volume containing $\sim 1.5 \times 10^{8} \mathrm{CFUs} / \mathrm{ml}$ P. aeruginosa was directly injected by one-shot injection in a single dose into the tumour, 13 days post-tumour implantation at its center area using the 1-cc 
insulin disposable syringe. The given dose was confirmed by plating bacterial dilutions on nutrient agar plates, and the bacteria were then counted by determining CFU in triplicate and expressed as $\mathrm{CFU} / \mathrm{ml}$.

\section{The exposure system}

The experimental setup was designed to be suitable for localized exposure protocol of the tumour mass only. The exposure device was fabricated and manufactured locally in the Biophysics Department research lab, Faculty of Science, Cairo University, Egypt. In this experiment the resonance frequency $0.7 \mathrm{~Hz}$ was used to control P. aeruginosa infection, according to previously reported by Serag et al. (2014).

Direct current power supply (15 V d.c.) with an electronic switching device was constructed to produce a square pulsed electrical current with an extremely low frequency (ELF) of range $0.1-20 \mathrm{~Hz}$. The pulsed electric currents were allowed to pass through a solenoid (magnetic gun), to generate magnetic pulses. The gun was of $1 \mathrm{~mm}$-thick copper wire wound around a ferromagnetic bar of $1 \mathrm{~cm}$-diameter. The tip of the gun was set $2 \mathrm{~cm}$ away perpendicular to the implanted tumour. The magnetic flux density (B) was $0.88 \pm 0.02 \mathrm{mT}$ at this distance as measured by a Gauss/Tesla meter (model 4048, manufactured by F.W. Bell, with probe T-4048.001USA) of accuracy $\pm 2 \%$. During the exposure period, each animal was fixed from its back on a sterile wooden board using sticky rubber tapes, which were soft to avoid any pressure/stress on the animal.

The software program, Sigma Plot V 12.5 was utilized in order to analyze data of the induced magnetic field distribution in a horizontal plane at a distance $2 \mathrm{~cm}$ from the tip of the magnetic gun (Fig. 1) prior to performing the experiments on mice.
Mice tumour volume estimation, assessment of antitumour activity and determination of the survival time

Tumour growth in all the experimental groups was monitored from day 8 to 42 post-tumour implantation by measuring the tumour volume with a digital caliper of accuracy \pm $0.01 \mathrm{~mm}$ (Tricle Brand, Shanghai, China). Caliper measurements were taken regularly for the 3 mutually orthogonal tumour diameters. The tumour volume $V$ was calculated using the following equation as suggested by Ning et al. (1994):

$V\left(\mathrm{~cm}^{3}\right)=\frac{\pi}{6} \times L W H$

where $L, W$, and $H$ are the length, width, and height of the tumour mass, respectively.

All mice groups were monitored clinically by daily observing the general health status, and the survival rates of mice with time for all groups until spontaneous death were determined.

At day 5 post-treatment, 10 mice from each group were sacrificed by cervical dislocation. Tumour tissues were dissected from three sacrificed mice from each group and processed for ultra-structural examinations. Then, the lung tissue samples were prepared for histopathology. For the other sacrificed animals, tumour masses which were complete excised from 3 mice in each group were frozen on dry ice and processed for flow cytometry analysis. In addition, bulk tumours from the remaining sacrificed 4 mice from each group were taken immediately for dielectric relaxation studies.

\section{Histopathological examination}

Lungs were carefully dissected out after sacrificing the mice, then fixed in $10 \%$ neutral formalin solution, after that pro-
A

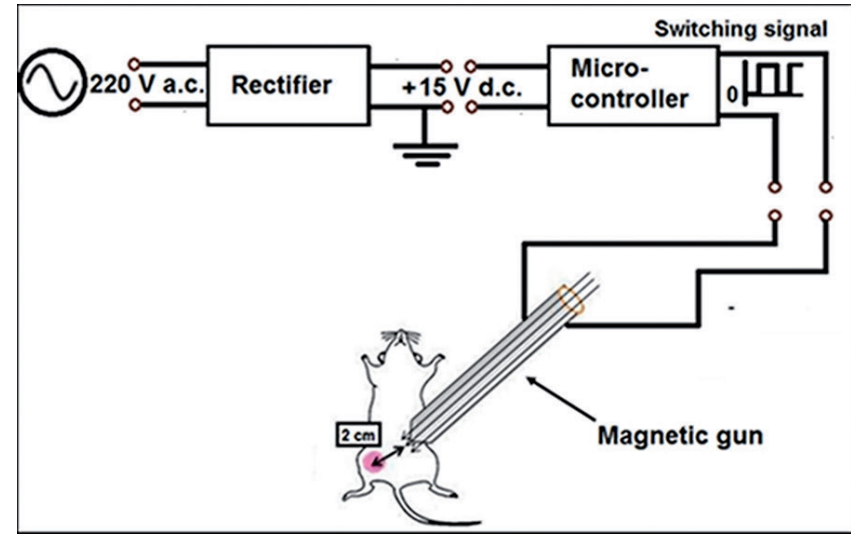

B

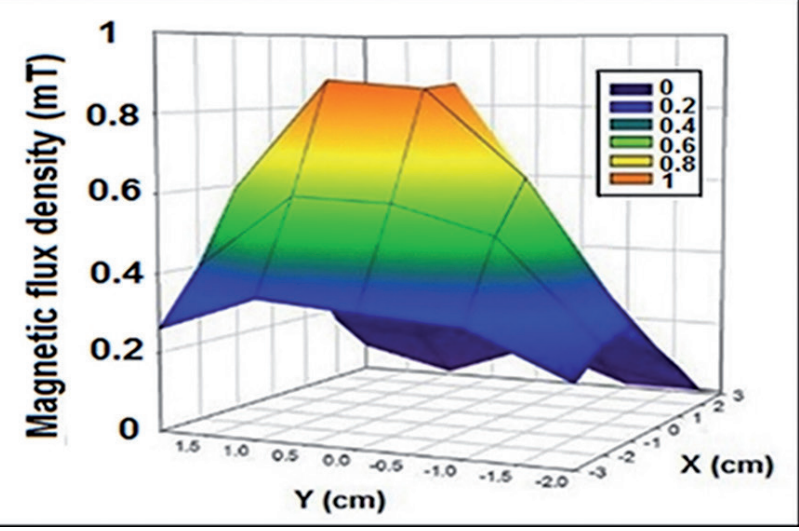

Figure 1. A. The schematic diagram of the experimental magnetic pulses-generating exposure system. B. Graphical representation of the 2 -D distribution of magnetic flux density $(\mathrm{mT})$ at a distance $2 \mathrm{~cm}$ from the tip of the magnetic gun. The peak flux density was $0.88 \pm 0.02 \mathrm{mT}$. 
cessed for standard histological techniques with Hematoxylin-Eosin (H\&E) stain (Bancroft and Gamble 2002), and examined under a light microscope (CX 31 Olympus optic microscope, Tokyo, Japan) connected with a computercontrolled digital camera (Canon).

Ultra-structural characteristics under Transmission Electron Microscope (TEM)

Briefly, tumour samples from each group were cut into $\sim 1 \mathrm{~mm}^{3}$ fragments and fixed by immersion in $2.5 \%$ glutaraldehyde for $1 \mathrm{~h}$ at $4^{\circ} \mathrm{C}$ in refrigerator and fixed again with $1 \%$ osmium tetroxide for $30 \mathrm{~min}$. Following that, samples were dehydrated with gradient series of alcohol and then embedded in epoxy resin. Thin sections were cut using an automatic Ultracut $S$ ultramicrotome, then stained with tolodin blue (1X) $5 \mathrm{~min}$, and examined by light microscope. Ultra thin sections were then prepared at a thickness approximately 75-90 $\mu \mathrm{m}$ and placed on 300-mesh, copper grids, routinely double stained with uranyl acetate and lead citrate to enhance. Finally, the prepared sections were examined and the images were captured by CCD camera model AMT using the TEM JEOL (JEM-1400 TEM).

Cell cycle study of tumour cells by flow cytometric analysis

The flow cytometric analysis was performed in the National Cancer Institute (NCI), Cairo, Egypt. Each frozen tumour mass was separately cut into small pieces from all sides, rinsed with PBS (10 min, $13000 \mathrm{rpm}$ ) to obtain $\sim 10^{6}$ singlecell suspensions. The cells from each tumour sample within the group were collected in 3 different centrifuge tubes, then each tube rinsed twice in PBS and fixed in a permeabilization solution of $70 \%$ ice-cold ethanol for 5 min then again washed with PBS. Cell pellets re-suspended in $250 \mathrm{ml}$ of trypsin solution, left $10 \mathrm{~min}$ at room temperature (20 to $25^{\circ} \mathrm{C}$ ), and then $200 \mathrm{ml}$ of the neutralizing solution (tripsin inhibitor and RNase buffer) was added to each tube and leave it again $10 \mathrm{~min}$ at room temperature. Prior to analysis, $200 \mathrm{ml}$ of cold $\left(2\right.$ to $\left.8^{\circ} \mathrm{C}\right)$ propidium iodide (PI) stain solution was added to each tube, and the cells were incubated for $10 \mathrm{~min}$ in the dark in a refrigerator. Thereafter, about 30,000 single tumour cells were scanned for DNA cell cycle distribution by a FACSCalibur flow cytometry system (FACScan; Becton Dickinson, San Jose, CA, USA) (Vindelov 1977), and the outcomes were expressed as cellular DNA content histograms for each sample. The average values at each phase of the cell cycle were calculated across samples within the groups.

\section{Dielectric measurements for Ehrlich tumour mass tissue}

The bulk tumours usually came out as a one piece and the entire mass of the tumour tissue was used for dielectric measurements within just about 10 min after tumour tissue removal from each animal's thigh so as to prevent dryness of the tissue keeping its activity as a living tissue in the measurements. A pair of $1 \mathrm{~cm}$ diameter stainless steel circular electrodes was set while the tumour sample was filling the whole volume in a special glass test cell between those electrodes. The separation distance between the electrodes was adjusted to provide excellent ohmic contact with a constant pressure, according to the tumour size, and this distance was measured with a micrometer.

Dielectric measurements were run in the frequency range from $1-500 \mathrm{kHz}$ by means of a programmable automatic RLC bridge (model Hioki, 3532, LCR Hi Tester, version 1.02,1999, Japan). The equivalent circuit for a biological sample placed between two electrodes (Schwan 1963) is a capacitance $\left(C_{S}\right)$ in parallel with a resistance $\left(R_{S}\right)$ representing the biological sample polarization, in series with a capacitance $\left(C_{P}\right)$ and a resistance $\left(R_{P}\right)$ in series representing the electrode polarization. The total capacitance $\left(C_{T}\right)$ and the total resistance $\left(R_{T}\right)$ of the equivalent circuit are usually measured as a parallel $\mathrm{RC}$ combination and recorded at different frequencies in the mentioned range.

The capacitance of the sample $C_{S}$ is given by:

$C_{S}=\varepsilon_{o} \varepsilon^{\prime} \frac{A}{d}$

where $\varepsilon_{0}$ is the permittivity constant of free space $(8.85$ $\left.\times 10^{-12} \mathrm{~F} / \mathrm{m}\right), \varepsilon^{\prime}$ is the relative permittivity of the tumour sample (dielectric constant), $A$ is the surface area of the electrode (in $\mathrm{m}^{2}$ ), and $d$ is the separation distance between the two electrodes (in meters).

The resistance of the biological sample $R_{S}$ is defined by the equation (Polk and Postow 1996):

$$
R_{S}=\frac{d}{\sigma A}
$$

where $\sigma$ is the total conductivity $(\mathrm{S} / \mathrm{m})$ of the sample (i.e., a.c. conductivity and d.c. conductivity of the sample).

In practice, the raw impedance data obtained are not indicative of true dielectric properties of the tumour sample where the data are affected by the electrode polarization. Thus, the relative permittivity $\varepsilon^{\prime}$ and the total conductivity $\sigma$ of the tumour sample are fitted to a single relaxation ColeCole dispersion model which contains the static ionic conductivity term $\sigma_{S}$ (Cole and Cole 1941; Gabriel et al. 1996):

$\varepsilon^{\prime}(f)=\frac{\Delta \varepsilon^{\prime}\left[1+\left(f / f_{c}\right)^{(1-\alpha)} \operatorname{Sin}(\alpha \pi / 2)\right]}{1+\left(f / f_{c}\right)^{2(1-\alpha)}+2\left(f / f_{c}\right)^{(1-\alpha)} \operatorname{Sin}(\alpha \pi / 2)}+\varepsilon_{\infty}$

$\sigma(f)=\frac{2 \pi f \varepsilon_{0} \Delta \varepsilon^{\prime}\left[\left(f / f_{C}\right)^{(1-\alpha)} \operatorname{Cos}(\alpha \pi / 2)\right]}{1+\left(f / f_{C}\right)^{2(1-\alpha)}+2\left(f / f_{C}\right)^{(1-\alpha)} \operatorname{Sin}(\alpha \pi / 2)}+\sigma_{s}$

where $\Delta \varepsilon^{\prime}$ is the dielectric increment where the dielectric constant $\varepsilon^{\prime}$ falls from high value $\varepsilon^{\prime}{ }_{S}$ to low value $\varepsilon^{\prime}{ }_{\infty}$ as the 
frequency increases through the dispersion region and was calculated by:

$\Delta \varepsilon^{\prime}=\varepsilon^{\prime}{ }_{s}-\varepsilon_{\infty}^{\prime}$

where $f_{c}$ is the critical frequency in $\mathrm{kHz}$, corresponding to the midpoint of the dispersion curve, and $\alpha$ is the distribution parameter which is related to width of the relaxation region.

The capacitance $C_{P}$ and the resistance $R_{P}$ of the electrode polarization were also fitted to a theoretical electrode polarization model. These experimental data give very good fit to the theoretical models using a nonlinear least-square genetic algorithm fitting program and the dielectric parameters $\varepsilon^{\prime}{ }_{\infty}$, $\Delta \varepsilon^{\prime}, f_{\mathcal{c}}, \sigma_{\mathrm{S}}$ and $\alpha$ are obtained for each tumour mass. Two fitting parameters $\left(\Delta \varepsilon^{\prime}\right.$ and $\left.\sigma_{S}\right)$ were useful for following changes in the cell membrane of tumour cells.

The average of the dielectric parameters of the four tumour samples is calculated in each group. The dielectric dispersion curve for each group was plotted by substituting the average parameter values in the theoretical Cole-Cole model for relative permittivity $\varepsilon^{\prime}$ and total conductivity $\sigma$ (Eq. 4 and 5) at different frequency points in the previously specified range.

\section{Statistical analysis}

The experimental results are expressed as mean values \pm standard errors of the means (S.E.M.). One-way analysis of variance (ANOVA) followed by least significance difference (LSD) test was applied to compare data differences between control and treated groups. A difference was considered statistically significant at probability value $p<0.05$. All these statistical analyses were done using the statistical SPSS (Statistical Product and Service Solutions) software of version 16.0 for Windows. The statistical analysis for mouse survival studies was performed by the Kaplan-Meier method using the MedCalc of 15.8 version, and the significance of differences comparing the survival curves among groups was assessed by the use of the Log-rank (Mantel Cox) test.

\section{Results}

\section{Morphological changes}

Some important morphological changes were observed on the tumour surfaces in the mice of GP2 as shown in Fig. 2. Within 4-8 days of starting the injection of bacteria within the core of the tumour, a black discoloration spot indicating an area of hemorrhagic necrosis developed on tumour surfaces. During the last 2 weeks of the experiment, this black spot expanded in size, then its color turned to be blue-greenish as a crust covering an ulcer. Another relevant observation was that, in some animals, the leg carrying the tumour began to dry, and then disappeared as illustrated in Figure 2C.

\section{Assessment of mice tumour volume}

Figure 3 represents the variation of mean tumour volume (in $\mathrm{cm}^{3}$ ) \pm S.E.M. as a function of days following tumour implantation over a 42-day period in different experimental groups.

At day 18 post-tumour implantation (i.e., day 5 posttreatment), the mean value of tumour volume of GP2 was $0.439 \pm 0.039 \mathrm{~cm}^{3}$, which was significantly lower than that of the GP1 and GP3 $\left(0.618 \pm 0.023 \mathrm{~cm}^{3}, p=0.001 ; 0.556 \pm\right.$ $0.027 \mathrm{~cm}^{3}, p=0.016$, respectively). At this time, GP4 showed a significant decrease in the tumour size $0.523 \pm 0.028 \mathrm{~cm}^{3}$ with respect to GP1 ( $p=0.044)$, but, not significantly with
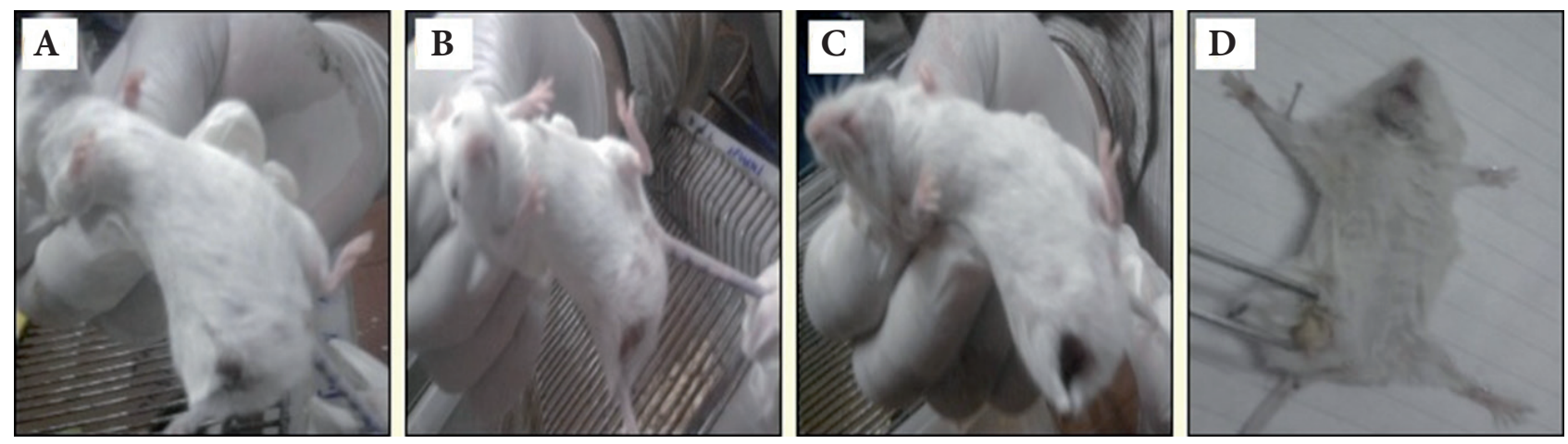

Figure 2. Examples of some abnormal noticeable signs seen under P. aeruginosa treatment of the mice of GP2. A. Black discoloration spot appeared on the tumour surface at the site of bacteria injection within 4-8 days of bacterial injection. B. The progress of aggressiveness of the bacterial infection, where the size of the black spot broader in size. C. The tumour-carrying leg of some mice began to dry and finally disappeared. D. The color of the spot occasionally turned to be blue-greenish as an abscess forming. 


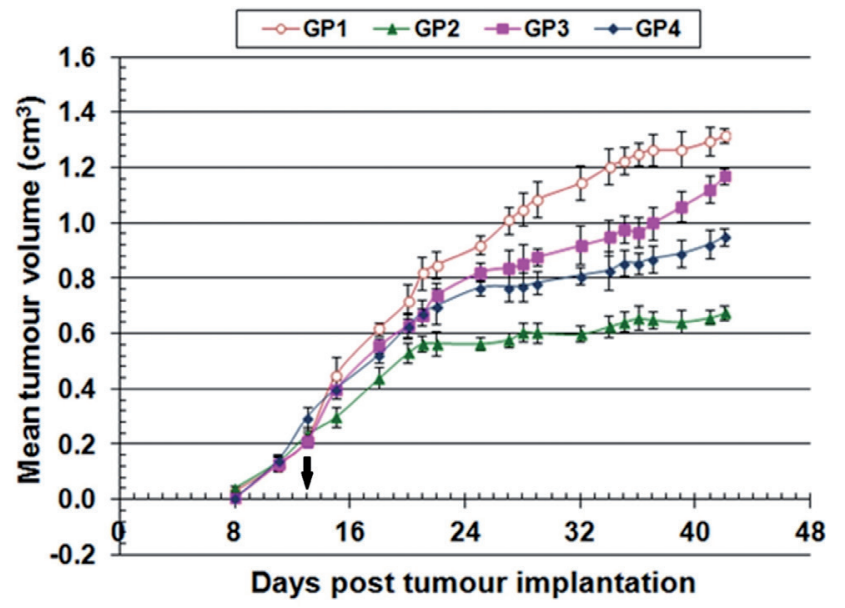

Figure 3. Time course of tumour growth curves and quantification of the antitumour efficacy of different treatments in Ehrlich tumour-bearing mice. Data are mean tumour volume \pm standard error of the mean (S.E.M.) for the surviving mice in each group. GP1, positive control tumour; GP2, tumour + P. aeruginosa; GP3, tumour + 0.7 Hz-SPMF; GP4, tumour + P. aeruginosa $+0.7 \mathrm{~Hz}-$ SPMF (the arrow indicates the day of starting treatment).

GP2 and GP3. On day 42 post-tumour implantation, significant decreases in the final tumour volume were detected in $\mathrm{GP} 2$ and GP4 $\left(0.675 \pm 0.024 \mathrm{~cm}^{3}\right.$, and $0.950 \pm 0.031 \mathrm{~cm}^{3}$, respectively; $p<0.0001$ in both) as compared to GP1 (1.314 \pm $\left.0.026 \mathrm{~cm}^{3}\right)$ and Gp3 $(p<0.0001, p=0.001$, respectively). Moreover, GP2 and GP4 showed significant differences with respect to each other $(p<0.0001)$. Whereas, in GP3,

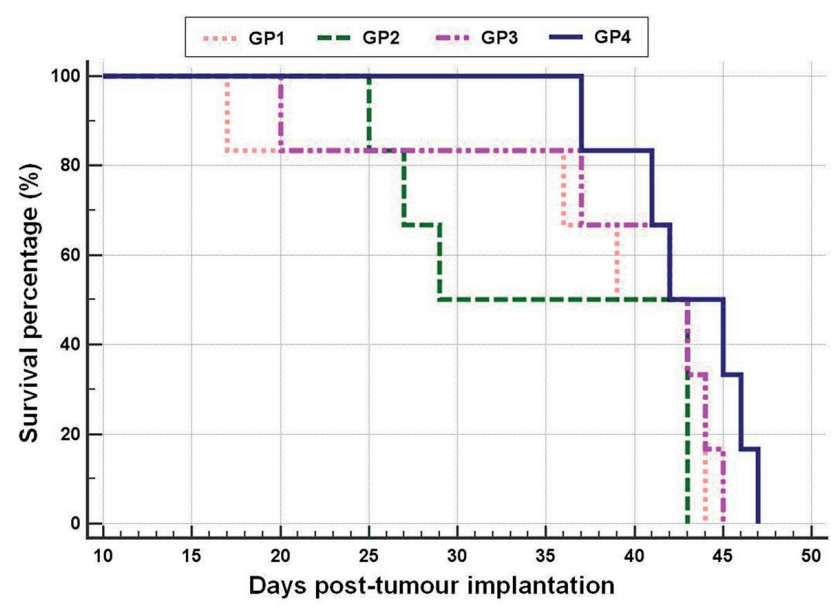

Figure 4. Kaplan-Meier survival curves until the spontaneous death of Ehrlich tumour-bearing mice of groups GP1 (positive control tumour), GP2 (tumour + P. aeruginosa), GP3 (tumour $+0.7 \mathrm{~Hz}-$ SPMF), and GP4 (tumour $+P$. aeruginosa $+0.7 \mathrm{~Hz}-\mathrm{SPMF}) . n=6$ mice/group; $p=0.225$, Log-rank test. final tumour volume reached an average value of $1.170 \pm$ $0.028 \mathrm{~cm}$, with $p=0.008$ when compared to the positive control group (GP1). The results clearly showed that mice belonging to GP2 exhibited the pronounced reduction in tumour growth rate compared to other groups.

\section{Survival of mice groups}

Figure 4 shows the comparison of the Kaplan-Meier survival curves of the variation of the survival percentage (\%) with the days following tumour implantation in different experimental groups. The curves showed non-significant differences in survival percentage between different mice groups ( $p=0.225$, using The Log-rank (Cox-Mantel) standard test, $n=6$ mice/group). It is obvious from the Figure 4 that the median survival time (MST, time of $50 \%$ survival) of GP2 was 29 days compared to 39 days for GP1. On the day 42 post- tumour implantation, the survival of the treated mice of GP3 and GP4 was found to be $50 \%$. It can be also noticed from Fig. 4 that mice of GP4 started to die on day 37 post-tumour implantation with $83.3 \%$ survival percent. On the same day GP1, GP2, and GP3 showed $66.7 \%, 50 \%$, and $66.7 \%$ survival, respectively. It is necessary to point that mice of GP2 had extremely high death rate and survived shorter than other experimental groups.

\section{Histopathological findings}

The microscopic examination of the lung tissues isolated from tumour bearing-mice of different experimental groups revealed various histopathological alterations (Fig. 5).

$\mathrm{H} \& \mathrm{E}$-stained lung sections of the GP1 demonstrated the appearance of lung metastases characterized mainly by proliferative changes in the bronchioles, including hyperplasia of bronchiolar epithelium and peribronchiolar infiltration with few tumour cells (Fig. 5A). Histopathological analysis of lung sections from mice of GP2 showed the same histopathological patterns over all sections, including focal interstitial pneumonia as shown in (Fig. 5B). For lung tissues from mice of GP3, only one section showed congestion of pulmonary blood vessels (Fig. 5C), whereas other sections of lungs from mice of this group revealed no detectable histopathological changes in lung architecture. In case of lung tissues from mice of GP4, there was focal atelectasis in some area, sometimes apparent (Fig. 5D). It is revealed that both treatments in GP2 and GP4 exerted toxicity in lung tissues of mice as a side effect, in particular, mice of GP2 were highly susceptible to severe lung infection.

\section{Transmission electron microscope (TEM) findings}

The transmission electron microscopic data of control Ehrlich tumour tissues from mice of GP1 (Fig. 6A) showed 

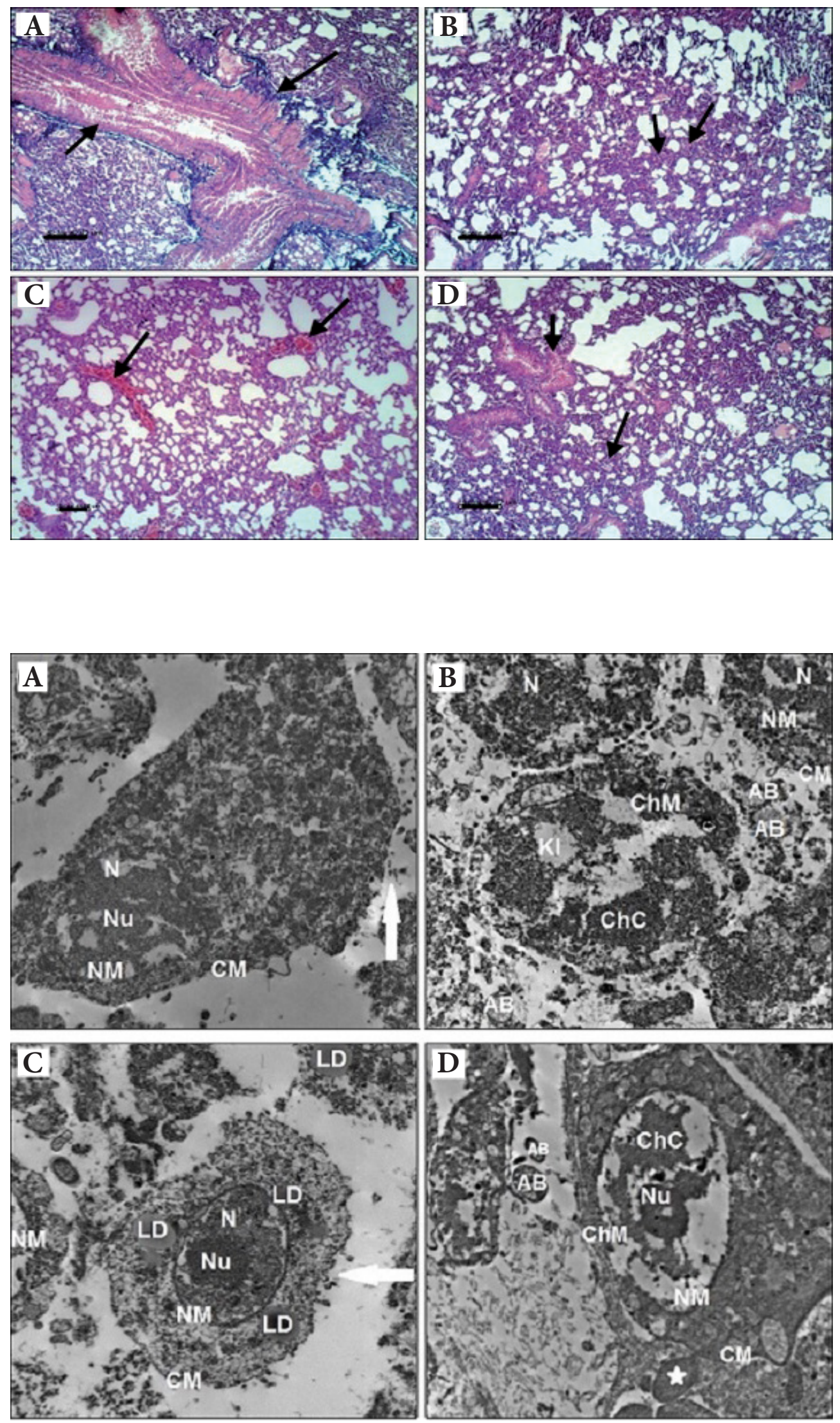

Figure 5. Lung histopathology photomicrographs of group GP1 (A) showing hyperplasia of bronchiolar epithelium and perivascular infiltration with few tumour cells (arrows), GP2 (B) showing signs of pneumonia (arrows), GP3 (C) showing congestion of pulmonary blood vessels (arrows) and GP4 (D) showing focal atelectasis (arrows), sometimes appeared. Magnification $\times 100$.
Figure 6. Ultrastructural photomicrographs of mice Ehrlich tumour cells of groups GP1 (A) showing normal elongated tumour cell structure, GP2 (B) showing late apoptotic tumour cell, GP3 (C) showing a tumour cell with lipid droplets (LD) within cytoplasm and GP4 (D) showing intermediate apoptotic tumour cell. Magnification $\times 8000$. N, nucleus; $\mathrm{Nu}$, nucleolus; $\mathrm{CM}$, cell membrane; NM, nuclear membrane; ChM, chromatin migration; ChC, chromatin condensation; $\mathrm{Kl}$, karyolysis; $\mathrm{AB}$, apoptotic bodies; white arrow pointing to microvilli; asterisk represents cell membrane blebs. 
normal intact elongated tumour cells without any obvious apoptotic morphology. As shown in the Figure 6A, the control tumour was characterized by round nuclear structure with evenly dispersed chromatin, and a chromatin migration was rarely detected in cells. There were clearly observed microvilli which are hair like structure features protruding on the cell surface and pointing to it by the white arrow in addition to that the nuclear membrane maintained its integrity. On the other hand, the treated tumour tissues of GP2 (Fig. 6B) exhibited clear significant changes in the shape of all tumour cells, including the typical morphological signs of programmed cell death (i.e., apoptosis). These apoptotic cells revealed irregular chromatin condensation into numerous clumps as dark aggregated masses with a clear separation between chromatins. In most instances, some cells exhibited distortion in the nucleus (i.e., karyolysis), with completely rupturing of the nuclear membrane associated with chromatin migration. Moreover, there was some alteration or damage in the cell membrane accompanied by loss of microvilli and formation of apoptotic bodies. For the SPMF-exposed tumour cells of GP3 (Fig. 6C), the general morphology and/or the arrangement of cell organelles were similar to that of control tumour cells. Nevertheless, there was a remarkable presence of large lipid droplets throughout the cell cytoplasm, more accurately located close to the nuclear membrane and the number of microvilli was decreased. In case of the treated tumour tissues of GP4 (Fig. 6D), there were tumour cells with apoptotic features too. A very small part of the nuclear membrane of some cells domed outward during which the chromatin was migrated; leaving most regions of the nucleus empty. Additional features include cell membrane blebbing and the cytoplasm containing swollen mitochondria.

\section{Effect of different treatments on Ehrlich tumour cell-cycle progression and apoptosis}

The DNA content histograms of positive control and of the three other treated Ehrlich tumours are presented in Figure 7. The treated tumours of GP2 revealed only a highly significant fraction in the Sub-G1 area, 5 days post-intratumoural bacterial injection (Fig. 7B); as compared with control tumours of GP1 where there were very few cells located in the Sub-G1 peak of the DNA histogram (only 7.97\% $\pm 1.20 \%$, Fig. 7A), which increased to $32.75 \% \pm 2.50 \%, p<0.0001$. However, the treated tumours of GP3 exhibited only a significant increase in the $\mathrm{G} 2 / \mathrm{M}$ phase cells from $1.06 \% \pm 0.35$ of control cells to $4.25 \% \pm 0.46 \%, p=0.012$ (Fig. 7E). By contrast, investigation of cell cycle distribution of treated tumours of GP4 (Fig. 7D) clearly showed a highly significant accumulation of the mean cells in $\mathrm{G} 2 / \mathrm{M}$ phase with respect to that of the other groups (Fig. 7E). The percentage of cells blocked in G2/M phase were $1.06 \% \pm 0.35 \%$ in control tumours and significantly ( $p<0.0001$ ) increased to $9.17 \% \pm 1.10 \%$ in these treated tumours of GP4. Moreover, this treatment (P. aeruginosa + $0.7 \mathrm{~Hz}$-SPMF) significantly increases the mean percentage of tumour cells in the Sub-G1 peak to $17.96 \% \pm 1.27 \%$ compared with $7.97 \% \pm 1.20 \%$ in the control group, $p=0.002$. It can be noticed from (Fig. 7E) that treated tumours of GP4
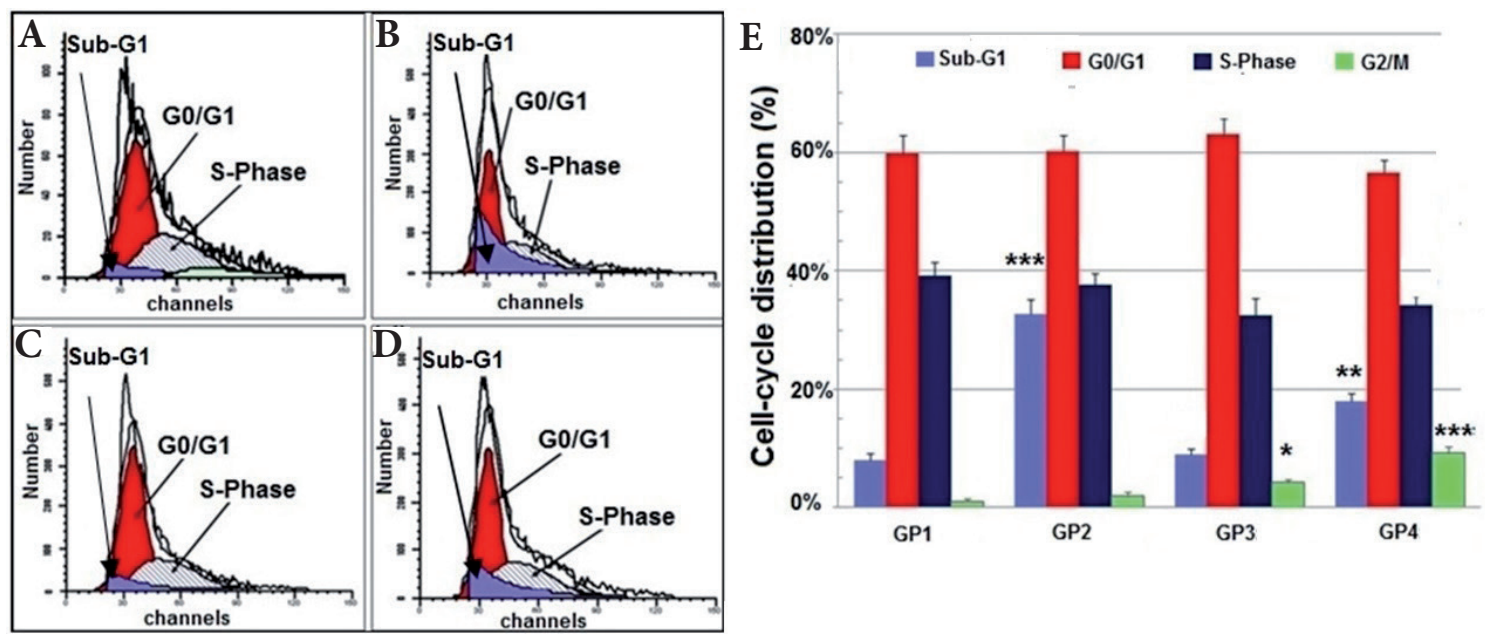

Figure 7. Cell-cycle distribution histograms obtained by flow cytometric analysis of positive control Ehrlich tumour of GP1 group (A), treated Ehrlich tumour of GP2 (B), GP3 (C), and GP4 (D) groups. E. The mean percentage of Ehrlich tumour cells in each phase of the cell cycle (Sub-G1, Go/G1, S, and G2/M) obtained by flow cytometry, comparing the different treatments to control. Each bar represents means of 3 tumour samples $(n=3)$ from each group \pm S.E.M. ${ }^{* * *},{ }^{* *}$, and ${ }^{\star}$ denote the difference which is statistically very high significant $(p<0.0001)$, high significant $(p<0.01)$, significant $(p<0.05)$, respectively, compared with control. NS, non-significant. 
exhibited much less Sub-G1 cell ratio than that of GP2, with significant difference $p<0.0001$.

Effect of different treatments on dielectric properties of the Ehrlich tumour

The mean values of the tumour tissue dielectric parameters: $\Delta \varepsilon^{\prime}$ and $\sigma_{S}$ for all the groups are listed in Table 1. The differences in the mean values of $\Delta \varepsilon^{\prime}$ and $\sigma_{S}$ between control tumours of GP1 and treated tumours of GP3 are not significantly different.

Figure 8 illustrates plots of variation of the frequencydependent relative permittivity (dielectric constant) $\varepsilon^{\prime}$ plotted on the left y axis, and the total electric conductivity $\sigma(\mathrm{S} / \mathrm{m})$ plotted on the opposite vertical axis versus the frequency range $(1-500 \mathrm{kHz})$ for the fresh tumours excised from mice ( $n=4$ /group) of all the experimental groups. Standard error bars are omitted for clarity. In general, it is clear from Figure 8 that there is an overall increase in the conductivity of tumour samples of all experimental groups with increasing frequency, while the relative permittivity decreases, showing a mirror image with each other, which
Table 1. Dielectric fitted Cole-Cole parameters for Ehrlich tumours excised from thigh of control mice and three different treated mice groups

\begin{tabular}{lcl}
\hline Group & \multicolumn{1}{c}{$\Delta \varepsilon^{\prime}$} & \multicolumn{1}{c}{$\sigma_{\mathrm{S}}(\mathrm{S} / \mathrm{m})$} \\
\hline GP1 & $2171.9 \pm 126$ & $0.47 \pm 0.03$ \\
GP2 & $370.5 \pm 18.94^{\star * *}$ & $0.72 \pm 0.04^{* * *}$ \\
GP3 & $1926 \pm 102.4(\mathrm{NS})$ & $0.47 \pm 0.02(\mathrm{NS})$ \\
GP4 & $886.5 \pm 50.3^{\star *}$ & $0.54 \pm 0.02(\mathrm{NS}=0.14)$ \\
\hline
\end{tabular}

Each parameter value represents mean \pm S.E.M. for 4 tumour samples $(n=4)$. GP1 group, positive control tumour; GP2 group, tumour+P. aeruginosa; GP3 group, tumour+0.7 Hz-SPMF; GP4 group, tumour $+P$. aeruginosa $+0.7 \mathrm{~Hz}-\mathrm{SPMF} ; \Delta \varepsilon^{\prime}$, dielectric increment; $\sigma_{S}$, the static (d.c.) ionic conductivity; NS, not significant difference from control; ${ }^{* *}$ highly significant difference from control $(p<$ $0.01) ;{ }^{* *}$ very highly significant difference from control $(p<0.0001)$.

means that the dielectric measurements met the KramersKronig requirement (Foster and Schwan 1989).

It is distinctly observed that the dielectric behavior of treated tumours from treated GP3 is approximately similar to that from control group (GP1).
A

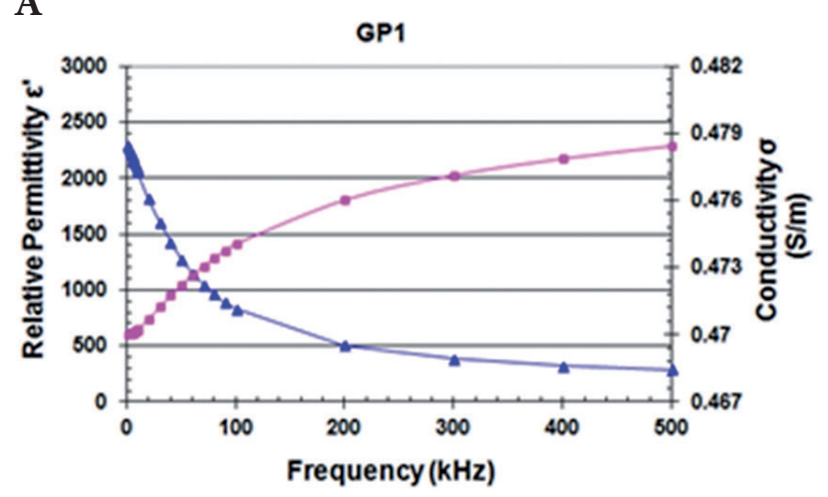

C

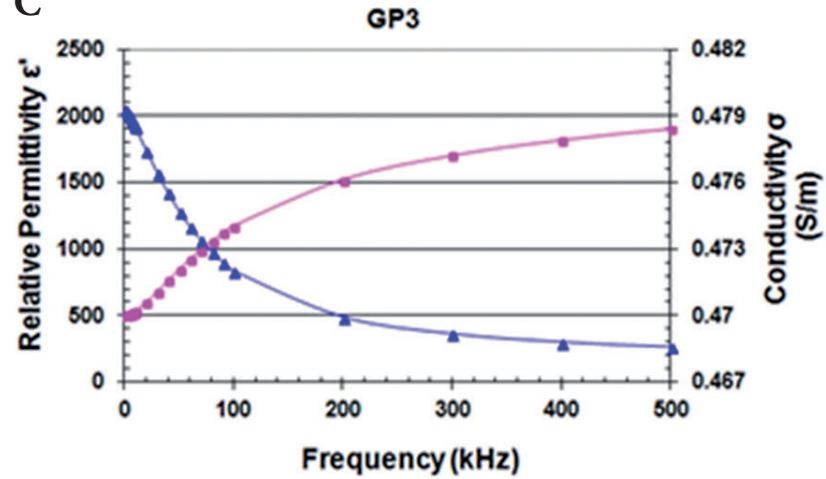

B

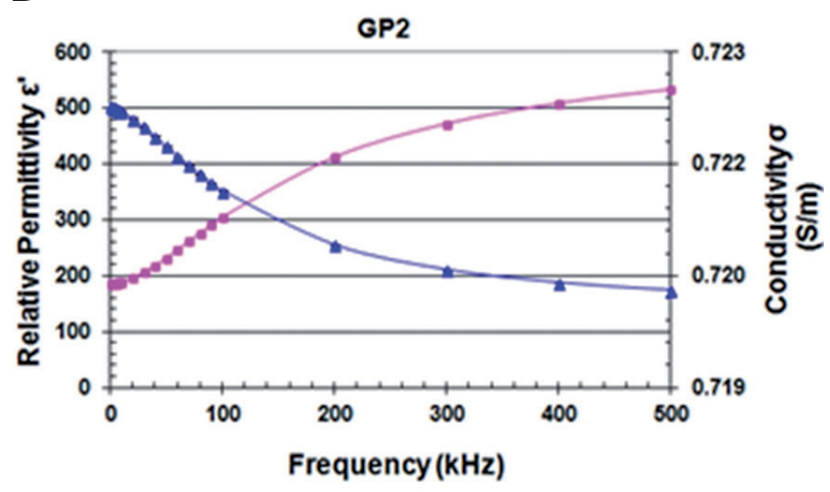

D

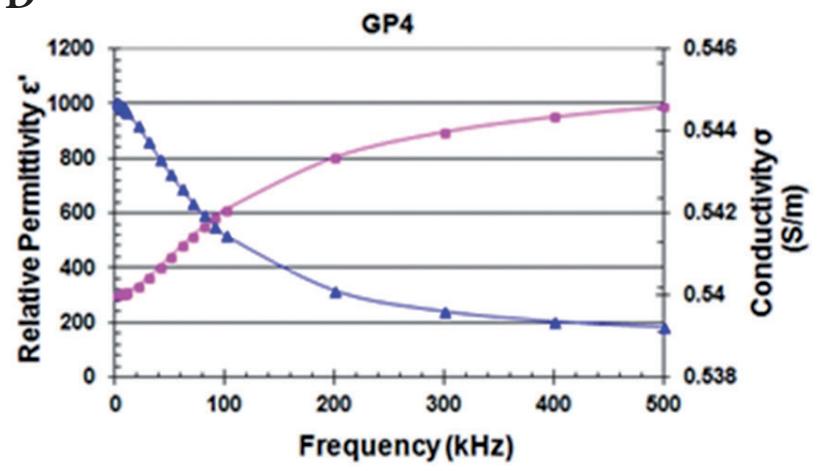

Figure 8. The variation of the mean relative permittivity $\varepsilon^{\prime}(-)$, and the total electric conductivity $\sigma(\star)$ as a function of the applied frequency over the range $1-500 \mathrm{kHz}$ at $37 \pm 0.2^{\circ} \mathrm{C}$ for fresh tumour tissues excised from mice of GP1 (positive control tumour, A), GP2 (tumour + P. aeruginosa, B), GP3 (tumour + 0.7 Hz-SPMF, C) and GP4 (tumour + P. aeruginosa + 0.7 Hz-SPMF, D) groups. 


\section{Discussion}

From the present work one can estimate that, live bacteria produce significant toxicity and side effects as reported by Jain and Forbes (2001) and Dang et al. (2001), thus their clinical use has been limited. This is in agreement with the present results where GP2 exhibited the high mortality rate (Fig. 4). Thus, the resultant mortality may be due to the secretion of robust lethal toxins from $P$. aeruginosa bacteria colonizing within the tumours. Moreover, abnormal noticeable signs seen in mice of GP2 (Fig. 2) are similar to that recorded by Dang et al. (2001) who noticed an extensive hemorrhagic necrosis area developed on tumour surfaces with black spot when used a combination of bacteriolytic therapy (COBALT), with usual chemotherapeutic drugs. Additionally, these toxicity results were verified by the histopathological examinations of lung sections. The lung tissue of GP2 showed focal interstitial pneumonia; five days post treatment (Fig. 5B). Such pneumonia signs may be the reason for the high mortality rate observed in mice of this group. These results were in context with Bryan and Reynolds (1984), Sadikot et al. (2005), Koenig and Truwit (2006), who reported that $P$. aeruginosa is a standout amongst the most widely recognized causes of life-threatening nosocomial-acquired pneumonia.

By contrast, the lung sections of GP4 revealed there was focal atelectasis in some area (Fig. 5D). Based on these histopathological findings, treated mice of GP4 develop minor bacterial loads in the lungs than GP2 mice treated by intratumoural injection of $P$. aeruginosa alone. Thus, exposure to $0.7 \mathrm{~Hz}$-SPMF might be beneficial in limiting the severity of infection. This was relatively obvious as indicated by what was acquired from survival results (Fig. 4). In spite of the fact that treatment of GP4 revealed no significant survival advantage they demonstrated a pattern toward reduction death rate.

The profiles of the tumour volumes under the employed different treatments as compared with control tumour can be investigated by the ultrastructural studies of tumour tissues, 5 days following treatment. TEM evidenced that both treated tumours of GP2 and GP4 have clearly visible ultra-morphological features of apoptosis (Fig. 6B, D). These apoptotic features are supported according to those reported by Weinrauch and Zychlinski (1999) and Iordache et al. (2011) who reported that bacteria generally have evolved several ways to trigger host cell apoptosis, including the secretion of protein synthesis inhibitors and activation of apoptotic proteins such as caspases that are cell cycle specific, pore forming proteins for surviving intracellularly by escaping phagosomes and molecules for the activation of host endogenous death mechanism in the infected/injected cells. In particular, both the extrinsic caspase-dependent and intrinsic mitochondria-dependent apoptosis pathways are provoked by live $P$ aeruginosa bacteria as reported by Jendrossek et al. (2001) and Cannon et al. (2003), that may explain these ultra-structural changes in Ehrlich tumour cells of both GP2 and GP4.

Visualization of more cells underwent apoptosis was evident in GP2 as compared with that of GP4, which may be attributed to the persistence of $P$. aeruginosa bacterial colonies growing in the tumour site of GP2; enable bacteria to continuously efflux various toxic proteins for an unlimited period of time.

These apoptotic observations detected in treated tumour cells of both GP2 and GP4 were further confirmed by flowcytometric analyses for cell cycle of Ehrlich tumour cells, where the nuclei of apoptotic cells contain hypo-diploid amount of DNA and can be recognized by their diminished susceptibility to staining with PI that was represented by measuring the degree of increase in the cell percentage of Sub-G1 peak (i.e., < 2n DNA content) in the DNA frequency histogram (Nicoletti et al. 1991; Darzynkiewicz et al. 1992). The results showed the significant highest percentage of apoptotic tumour cells evidenced by the appearance of a progressively larger Sub-G1 peak $(32.75 \% \pm 2.5 \%)$ in P. aeruginosa-treated Ehrlich tumour of GP2 (Fig. 7B and E) in comparison with the control and other treated tumour cells. Also, there was a significant increase in apoptosis percentage, but with only $(17.96 \% \pm 1.27 \%)$ as identified from the Sub-G1 in DNA histogram of treated Ehrlich tumour cells of GP4 (Fig. 7D and E), as compared with control tumour cells of GP1. Based on the preceding results and their interpretations, the antitumour activity of both treatments in GP2 and GP4 is associated with induction of apoptosis. Apoptosis induction of $P$. aeruginosa bacterium can be managed by producing several cytotoxic products inside the tumours; for example Azurin protein (Yamada et al. 2002, 2004) and P. aeruginosa exotoxin A (ETA) (Keppler-Hafkemeyer et al. 2000; Schmidt et al. 2001), as well as the activity of this toxin as an immunotoxin has been reported (Lorberboum-Galski 2011; Weldon and Pastan 2011).

Conversely, the apoptotic (Sub-G1) peak was not clearly detectable in the histogram of $0.7 \mathrm{~Hz}$-SPMF-exposed tumour cells of GP3, without significant difference to that of control cells (Fig. 7C and E). This result is in harmony with the TEM observations, where treated Ehrlich tumour cells of GP3 did not exhibit any obvious apoptotic features (Fig. 6C); somewhat like the positive control Ehrlich tumour cell of the GP1 as displayed in (Fig. 6A). The only obvious difference was that tumour cell of GP3 showed remarkable presence of large lipid droplets dispersed throughout the cell cytoplasm. Previous studies reported that cancer cells under stressful environments exhibited cytoplasmic lipid droplets formation (Delikatny et al. 2002; Iorio et al. 2005). Nevertheless, in the context of cell cycle, treatment with $0.7 \mathrm{~Hz}-\mathrm{SPMF}$ resulted in a significant increase of cell accumulation in the G2/M phase 
compared to control. This finding is in line with a previous study by Delle-Monache et al. (2013) that showed nearly the same result after exposure of specific endothelial cells to a sinusoidal MF $(2 \mathrm{mT}, 50 \mathrm{~Hz})$. The authors interpreted this arrest of cell cycle progression at the G2/M phase as a transiently delay in the mitosis phase that was partially recovered during time, then the cell cycle duration can return to its default state. Indeed, the data about the effect of ELFEMF on the cell cycle is contradictory and this difference is surely due to variations in the (e.g., exposure condition, cell type used, age, etc.). According to the exposure system parameters (exposure pattern and exposure time) identified in this study, GP3 has had largest tumour volume among the two other treatment groups as displayed in Figure 3.

Interestingly, as can be seen from the DNA histogram (Fig. 7D) of treated Ehrlich tumour cells of GP4, a significant accumulation of cells in the G2/M phase was also clearly observed compared to control tumour cells (Fig. 7E). In fact, the tumour cells of GP4 had a large population of G2/M phase arrest cells than tumour cells of GP2. Collectively, it could be deduced that two major processes occur at the cellular level involved in tumour regression of GP4: the P. aeruginosa bacteria were initially shown to create antitumour effects through induction of apoptosis that accompanied with temporary (reversible) delay of the cell cycle progression at the G2/M phase exerted by $0.7 \mathrm{~Hz}-\mathrm{SPMF}$ exposure.

It is noteworthy that the results of dielectric properties are in a distinct correlation with tumour cells that underwent apoptosis, which means that the dielectric property trends of tumour tissues can be also used as an indicator for treatment effectiveness. From the results shown in Figure 8, it can be noticed that all studied tumour samples (control and treated) exhibit one distinctly dielectric dispersion at the specified frequency range of $1-500 \mathrm{kHz}$, which is known as the $\beta$-dispersion. These $\beta$-dispersion phenomena are arising mainly from interfacial polarizations of cellular membranes (Schwan 1957; Foster and Schwan 1994). Where, the plasma membrane is considered among the many features that can discriminate between living and non-living cells (van Engeland et al. 1997). From the results obtained in this work for dielectric parameters of tumour tissues under different treatments (Table 1), it is clear that the treated tumours of GP2 exhibited a noticeable significant increase $(p<0.0001)$ in the mean value of the static (d.c.) ionic conductivity $\sigma_{S}$, as compared with the control tumours. This resulting increase in $\sigma_{S}$ may be due to that the treatment employed for GP2 induced a sharp rise in the leakage of ions in the extracellular fluid of cells. The reason for this detected ionic leakage may be attributed to that the membranes of tumour cells become strongly injured during bacterial infection, resulting in mixing the intracellular fluid ions with that of the extracellular fluid and hence contributing to the static conductivity. This finding is in line with the scientific logic due to the ability of
P. aeruginosa bacteria to secrete pore forming proteins PFPs (toxins) that disrupt membrane lipid bi-layers integrity of the target cells by creating pores (Bischofberger et al. 2009). These cellular membranes became highly permeable as there was a continuous production of PFPs during infection with $P$. aeruginosa, also loosed its ability to hold electrical charges (i.e., capacitance). Another explanation could be contributed to that decrease in capacitance is surface morphology destruction (i.e., microvilli) as shown in the TEM of GP2 and GP4 (Fig. 6B and D). These missing structure features result in a decrease in membrane surface area and consequently in permittivity of tumour cells that with porous-membranes. All this reflects the collapse in the $\beta$-dispersion magnitude that can be represented by the dielectric increment $\Delta \varepsilon^{\prime}$. The mean values of dielectric increments for treated tumours of both GP2 $(p<0.0001)$ and GP4 $(p<0.01)$ were significantly lower than that for control tumours of GP1. Collectively, these mentioned changes in the dielectric properties of tumour samples indicated changes in the structure and shape of cellular membranes of tumour cells especially with progressed Pseudomonal-infection.

\section{Conclusion}

In this study, an intratumoural injection of $P$. aeruginosa combined with tumour local exposure to $0.7 \mathrm{~Hz}-\mathrm{SPMF}$ retarded the growth of Ehrlich tumour. It seems that $P$. aeruginosa bacteria were responsible for that antitumour activity because of its ability to secrete antitumour toxic proteins. Moreover, the strategy of using combined SPMF provides to some extent a safe therapeutic effect by reducing normal tissue injuries. Since the exposure procedure seems to serve as an important handling role to limit infection, further examination is needed to make this technique clinically valid.

Acknowledgement. The authors are grateful to Prof. Dr. Ali ElLakany, Professor of Biophysics, Faculty of Science, Cairo University for his help in the dielectric data analysis.

Conflict of interests. The authors report no conflict of interests. The authors alone are responsible for the content and writing of the paper.

\section{References}

Bancroft J. D., Gamble M. (2002): Theory and Practice of Histological Technique. (5th Ed.), Churchill Livingstone, Edinburg and London

Bischofberger M., Gonzalez M. R., van der Goot F. G. (2009): Membrane injury by pore-forming proteins. Curr. Opin. Cell Biol. 21, 589-595 https://doi.org/10.1016/j.ceb.2009.04.003 
Bryan C.S., Reynolds K. L. (1984): Bacterial nosocomial pneumonia analysis of 172 episodes from a single metropolitan area. Am. J. Respir. Crit. Care Med. 129, 668-671

Cannon C. L., Kowalski M. P., Stopak K. S., Pier G. B. (2003): Pseudomonas aeruginosa-induced apoptosis is defective in respiratory epithelial cells expressing mutant cystic fibrosis transmembrane conductance regulator. Am. J. Respir. Cell Mol. Biol. 29, 188-197 https://doi.org/10.1165/rcmb.4898

Chakrabarty A. M. (2003): Microorganisms and cancer: Quest for a therapy. J. Bacteriol. 185, 2683-2686 https://doi.org/10.1128/JB.185.9.2683-2686.2003

Cole K. S., Cole R. H. (1941): Dispersion and adsorption in dielectrics: I. Alternating current characteristics. J. Chem. Phys. 9, 341-351 https://doi.org/10.1063/1.1750906

Dang L. H., Bettegowda C., Huso D. L., Kinzler K. W., Vogelstein B. (2001): Combination bacteriolytic therapy for the treatment of experimental tumors. Proc. Natl. Acad. Sci. USA 98, 15155-15160 https://doi.org/10.1073/pnas.251543698

Darzynkiewicz Z., Bruno S., Del Bino G., Gorczyca W., Hotz M. A., Lassota P., Traganos F. (1992): Features of apoptotic cells measured by flow cytometry. Cytometry 13, 795-808 https://doi.org/10.1002/cyto.990130802

Delikatny E. J., Cooper W. A., Brammah S., Sathasivam N., Rideout D. C. (2002): Nuclear magnetic resonance-visible lipids induced by cationic lipophilic chemotherapeutic agents are accompanied by increased lipid droplet formation and damaged mitochondria. Cancer Res. 62, 1394-1400

Delle-Monache S. D., Angelucci A., Sanità P., Iorio R., Bennato F., Mancini F., Gualtieri G., Colonna R. C. (2013): Inhibition of angiogenesis mediated by extremely low-frequency magnetic fields (ELF-MFs). PLoS One 8, 1 https://doi.org/10.1371/journal.pone.0079309

Fadel M. A., Mohamed S. A., Abdelbacki A. M., El-Sharkawy A. H. (2014): Inhibition of Salmonella typhi growth using extremely low frequency electromagnetic (ELF-EM) waves at resonance frequency. J. Appl. Microbiol. 117, 358-365 https://doi.org/10.1111/jam.12527

Fadel M. A., Reem H. Elgebaly, Elneklawi M. S., Othman A. S. (2016): Role of duty cycle on Pseudomonas aeruginosa growth inhibition mechanisms by positive electric pulses. Bio-Med. Mater. Eng. 27, 211-225 https://doi.org/10.3233/BME-161577

Foster K. R., Schwan H. P. (1989): Dielectric properties of tissues and biological materials: a critical review. Crit. Rev. Biomed. Eng. 17, 25-104

Foster K. R., Schwan H. P. (1994): Dielectric properties of tissues. In: Handbook of Biological Effects of Electromagnetic Fields. (Eds. C. Polk and E. Postow), Boca Raton, Fla, CRC Press

Gabriel S., Lau R. W., Gabriel C. (1996): The dielectric properties of biological tissues: III. Parametric models for the dielectric spectrum of tissues. Phys. Med. Biol. 41, 2271-2293 https://doi.org/10.1088/0031-9155/41/11/003

Iordache C., Bleotu C., Holban A., Lixandru M., Cotar A., Lazar V., Antohe F., Chifiriuc M. C. (2011): Differential effects on caspase mediated apoptosis of hela cells induced by different
Pseudomonas aeruginosa culture fractions. I. J. A. B. P. T. (India) 2, 132-138

Iorio E., Mezzanzanica D., Alberti P., Spadaro F., Ramoni C., D‘Ascenzo S., Millimaggi D., Pavan A., Dolo V., Canevari S., Podo F. (2005): Alterations of choline phospholipid metabolism in ovarian tumour progression. Cancer Res. 65, 9369-9376

https://doi.org/10.1158/0008-5472.CAN-05-1146

Jain R. K., Forbes N. S. (2001): Can engineered bacteria help control cancer? Proc. Natl. Acad. Sci. USA 98, 14748-14750 https://doi.org/10.1073/pnas.261606598

Jendrossek V., Grassme H., Mueller I., Lang F., Gulbins E. (2001): Pseudomonas aeruginosa-induced apoptosis involves mitochondria and stress-activated protein kinases. Infect. Immun. 69, 2675-2683

https://doi.org/10.1128/IAI.69.4.2675-2683.2001

Keppler-Hafkemeyer A., Kreitman R. J., Pastan I. (2000): Apoptosis induced by immunotoxins used in the treatment of hematologic malignancies. Int. J. Cancer 87, 86-94 https://doi.org/10.1002/1097-0215(20000701)87:1<86::AID-IJC13>3.0.CO;2-I

Koenig S. M., Truwit J. D. (2006): Ventilator-associated pneumonia: diagnosis, treatment, and prevention. Clin. Microbiol. Rev. 19, 637-657 https://doi.org/10.1128/CMR.00051-05

Koneman E. W., Allen S. D., Janda W. M., Schreckenberger P. C., Winn W. C. (1992): Antimicrobial susceptibility testing. In: Color Atlas and Text Book of Diagnostic Microbiology. (4th Ed.), pp. 624, 629-637, J. B. Lippincott Co., USA

Kuan Y. D., Lee C. H. (2016): Salmonella overcomes tumor immune tolerance by inhibition of tumor indoleamine 2, 3-dioxygenase 1 expression. Oncotarget 7, 374-385

Li M., Qu J. H., Peng Y, Z, (2004): Sterilization of Escherichia coli cells by the application of pulsed magnetic field. J. Environ. Sci. 16, 349-352

Lorberboum-Galski H. (2011): Human toxin-based recombinant immunotoxins/chimeric proteins as a drug delivery system for targeted treatment of human diseases. Expert Opin. Drug Deliv. 8, 605-621 https://doi.org/10.1517/17425247.2011.566269

Maletzki C., Linnebacher M., Kreikemeyer B., Emmrich J. (2008): Pancreatic cancer regression by intratumoural injection of live Streptococcus pyogenes in a syngeneic mouse model. Gut 57, 483-491 https://doi.org/10.1136/gut.2007.125419

McFarland J. (1907): Nephelometer: an instrument for estimating the number of bacteria in suspension used for calculating the opsonic index for vaccines. J.A.M.A. 14, 1176-1178 https://doi.org/10.1001/jama.1907.25320140022001f

Michl P., Gress T. M. (2004): Bacteria and bacterial toxins as therapeutic agents for solid tumors. Curr. Cancer Drug Targets 4, 689-702 https://doi.org/10.2174/1568009043332727

Nicoletti I., Migliorati G., Pagliacci M. C., Grignani F., Riccardi C. (1991): A rapid and simple method for measuring thymocyte apoptosis by propidium iodide staining and flow cytometry. J. Immunol. Methods 9, 139-271 https://doi.org/10.1016/0022-1759(91)90198-o 
Ning S., Macleod K., Abra R. M., Huang A. H., Hahn G. M. (1994): Hyperthermia induced doxorubicin release from longcirculating liposomes and enhanced their anti-tumor efficacy. Int. J. Radiat. Oncol. Biol. Phys. 29, 827-834 https://doi.org/10.1016/0360-3016(94)90572-X

Oncul S., Cuce E. M., Aksu B., Inhan Garip A. (2016): Effect of extremely low frequency electromagnetic fields on bacterial membrane. Int. J. Radiat. Biol. 92, 42-49 https://doi.org/10.3109/09553002.2015.1101500

Pawelek J. M., Low K. B., Bermudes D. (1997): Tumor-targeted Salmonella as a novel anticancer vector. Cancer Res. 57, 4537-4544

Polk C., Postow E. (1996): Hand Book of Biological Effects of Electromagnetic Fields. (2nd Ed.), pp. 99-107, CRC Press BocaRaton, New York

Sadikot R. T., Blackwell T. S., Christman J. W., Prince A. S. (2005): Pathogen-host interactions in Pseudomonas aeruginosa pneumonia. Am. J. Respir. Crit. Care Med. 171, 1209-1223 https://doi.org/10.1164/rccm.200408-1044SO

Schmidt M., McWatters A., White R. A., Groner B., Wels W., Fan Z., Bast R. C. (2001): Synergistic interaction between an antip185HER-2 pseudomonas exotoxin fusion protein [scFv(FRP5)ETA] and ionizing radiation for inhibiting growth of ovarian cancer cells that overexpress HER-2. Gynecol. Oncol. 80, 145-155 https://doi.org/10.1006/gyno.2000.6040

Schwan H. P. (1957): Electrical properties of tissues and cell suspensions. Adv. Biol. Med. Phys. 5, 147-209 https://doi.org/10.1016/B978-1-4832-3111-2.50008-0

Schwan H. P. (1963): Determination of biological impedances. Phys. Tech. Biol. Res. 6, 323-407 https://doi.org/10.1016/b978-1-4831-6743-5.50013-7

Segatore B., Setacci D., Bennato F., Cardigno R., Amicosante G. Iorio R. (2012): Evaluations of the effects of extremely lowfrequency electromagnetic fields on growth and antibiotic susceptibility of Escherichia coli and Pseudomonas aeruginosa. Int. J. Microbiol. 2012, 7 https://doi.org/10.1155/2012/587293

Serag N., Fadel M. A., Osoris W. G. (2014): Healing of injuries of guinea pig contaminated with Pseudomonas aeruginosa by $0.7 \mathrm{~Hz}$ square magnetic impulses (new method). Conference: Proceedings of the German Biophysical Society. (Germany) September 14-17, 2014

Strasák L., Vetterl V., Šmarda J. (2002): Effects of low-frequency magnetic fields on bacteria Escherichia coli. Bioelectrochemistry $\mathbf{5 5}, 161-164$
https://doi.org/10.1016/S1567-5394(01)00152-9

Theys J., Barbe S., Landuyt W., Nuyts S., Van Mellaert L., Wouters B., Anne J., Lambin P. (2003): Tumor-specific gene delivery using genetically engineered bacteria. Curr. Gene Ther. 3, 207-221 https://doi.org/10.2174/1566523034578357

van Engeland M., Kuijpers H. J., Ramaekers F. C., Reutelingsperger C. P., Schutte B. (1997): Plasma membrane alterations and cytoskeletal changes in apoptosis. Exp. Cell Res. 235, 421-430 https://doi.org/10.1006/excr.1997.3738

Vázquez-Rivera D., González O., Guzmán-Rodríguez J., Díaz-Pérez A. L., Ochoa-Zarzosa A., López-Bucio J., Meza-Carmen V., Campos-García J. (2015): Cytotoxicity of cyclodipeptides from Pseudomonas aeruginosa PAO1 leads to apoptosis in human cancer cell lines. Biomed. Res. Int. 2015, 1-9 https://doi.org/10.1155/2015/197608

Vindelov L. L. (1977): Flow microfluorometric analysis of nuclear DNA in cells from solid tumors and cell suspensions. A new method for rapid isolation and straining of nuclei. Virchows Arch. B Cell Pathol. 24, 227-242

Weinrauch Y., Zychlinski A. (1999): The induction of apoptosis by bacterial pathogens. Annu. Rev. Microbiol. 53, 155-187 https://doi.org/10.1146/annurev.micro.53.1.155

Weldon J. E., Pastan I. (2011): A guide to taming a toxin - recombinant immunotoxins constructed from pseudomonas exotoxin A for the treatment of cancer. FEBS J. 278, 4683-4700 https://doi.org/10.1111/j.1742-4658.2011.08182.x

Yamada T., Goto M., Punj V., Zaborina O., Chen M. L., Kimbara K., Majumdar D., Cunningham E., Das Gupta T. K., Chakrabarty A. M. (2002): Bacterial redox protein azurin, tumor suppressor protein p53, and regression of cancer. Proc. Natl. Acad. Sci. USA 99, 14098-14103 https://doi.org/10.1073/pnas.222539699

Yamada T., Hiraoka Y., Ikehata M., Kimbara K., Avner B. S., Das Gupta T. K., Chakrabarty A. M. (2004): Apoptosis or growth arrest: modulation of tumor suppressor p53's specificity by bacterial redox protein azurin. Proc. Natl. Acad. Sci. USA 101, 4770-4775 https://doi.org/10.1073/pnas.0400899101

Received: July 23, 2016

Final version accepted: October 13, 2016

First published online: May 4, 2017 